\title{
Los itinerarios profesionales en Biología: un ejemplo de formación académica orientada a la inserción profesional*
}

\author{
Professional pathways in Biology: an example of professionally-oriented \\ teaching
}

José Aramburu, Fèlix Bosch, Jorge Pérez, Mariano Sentí y Josep-Eladi Baños

Facultad de Ciencias de la Salud y de la Vida. Universitat Pompeu Fabra.

Uno de los retos de la Universidad es formar los profesionales necesarios para la sociedad en que está inserta. Este ajuste no es fácil de obtener debido a la rigidez de los planes de estudios oficiales y a las cambiantes necesidades sociales. Sin embargo, el próximo Espacio Europeo de Educación Superior tiene este empeño como uno de sus principales objetivos. En el presente artículo se presenta la experiencia de los itinerarios profesionales de la licenciatura en Biología de la Universitat Pompeu Fabra durante dos cursos académicos. Situados en el quinto curso del plan de estudios, permiten a los estudiantes una formación específica en uno de los ámbitos profesionales en los que pueden insertarse laboralmente. La experiencia muestra que, tras un período de uno o dos años de su graduación, el $83 \%$ de los egresados se encuentran realizando un trabajo remunerado, en la mayoría de los casos relacionado con la biología, mientras que un 9\% realizan otros estudios (no doctorado).

\footnotetext{
* La experiencia descrita en este artículo fue premiada con la distinción Jaume Vicens Vives a proyectos colectivos de calidad docente universitaria en su convocatoria de 2005. Estos galardones son concedidos por el gobierno de la Generalitat de Catalunya a propuesta del Departament d'Universitats, Recerca i Societat de la Informació (DURSI).
}

Correspondencia:

Josep-E. Baños

Facultad de Ciencias de la Salud y de la Vida

Universitat Pompeu Fabra

Dr. Aiguader 80

08003-Barcelona

tel. 935422950

e-mail: jbanos@imim.es
Sólo el 3\% de los graduados están buscando trabajo de forma activa. En conclusión, los itinerarios profesionales pueden constituir una estrategia educativa adecuada para permitir que los licenciados en Biología se inserten con éxito en ocupaciones laborales acordes con su formación.

Palabras clave: Educación, inserción laboral, biología, itinerarios profesionales.

One of the main challenges facing universities is to train professionals who possess the skills that society requires. This is difficult to achieve due to the rigidity of official curricula and the constantly changing needs of society. However, it remains one of the main goals of the Bologna process. This paper describes the experience of the professional pathways which form part of the biology degree offered by the Universitat Pompeu Fabra, over a period of two academic years. Professional pathways are an essential part of the fifth year syllabus and provide students with specific training in one of the professional settings in which they may eventually work. Our experience shows that $83 \%$ of graduates are currently working in biology-related posts, while an additional $9 \%$ are engaged in postgraduate studies (non-PhD programmes). Only 3\% of graduates are actively seeking employment. In summary, professional pathways may constitute a valid educational strategy for preparing biology graduates for successful professional activity in biology-related environments.

Key words: education, entering the job market, biology, professional pathways. 
Entre los objetivos del Espacio Europeo de Educación Superior se encuentra el acercar la formación académica que ofrecen los centros universitarios con las demandas sociales ${ }^{1}$. Esta adecuación ha sido considerada en algunos sectores como una "venta encubierta" de la Universidad a las empresas $^{2}$, caricaturizando el hecho de que uno de los principales objetivos de esta institución es preparar profesionales que puedan satisfacer las necesidades de la sociedad en diversos ámbitos, y las empresas no son precisamente el último de ellos.

El comentario del párrafo anterior tiene mucho que ver con la definición de los perfiles profesionales de los egresados. Aunque no resuelta completamente, esta situación está relativamente clara en muchos de los provenientes de los estudios de ciencias de la salud debido a que su función social es aparentemente evidente ${ }^{3-5}$. No parece difícil entender qué se espera de un médico, un diplomado universitario en enfermería, un farmacéutico o un fisioterapeuta, pues todos ellos desempeñan funciones bastante definidas. En otros estudios, sin embargo, las cosas distan de estar tan claramente definidas.

El caso paradigmático en el ámbito de las ciencias de la salud es el de la licenciatura en Biología. A pesar de que muchos biólogos trabajan en centros de investigación biomédica, en laboratorios clínicos o en empresas del ámbito sanitario ${ }^{6}$, nunca se ha definido con claridad el destino profesional específico de un biólogo. Sus estudios abarcan desde la zoología a la biotecnología, pasando por la fisiología vegetal o la ecología. La licenciatura en Biología se encuentra en muchos de sus ámbitos en estrecho contacto con la Farmacia, la Medicina o la Química. En tiempos recientes, la situación se ha complicado aún más con la aparición de estudios de Bioquímica, Biotecnología o Ciencias Ambientales, por sólo citar algunos. De nuevo, parece claro el cometido de un biotecnólogo pero el de un biólogo sigue siendo incierto. Existen pocas dudas de que esta incertidumbre desdibuja el perfil profesional de los licenciados en Biología y dificulta su inserción laboral tras la licenciatura.

En 1998 la Universitat Pompeu Fabra (UPF) empezó a impartir la licenciatura en Biología con una orientación distinta de las presentes en el resto de universidades de su entorno ${ }^{7,8}$. Algunas de ellas habían intentado corregir la situación descrita con la creación de segundos ciclos destinados a la especialización en diversos ámbitos. La UPF apostó por un nuevo modelo que mantenía una licenciatura de cinco años, cuatro de ellos comunes y un quinto des- tinado a los denominados itinerarios profesionales (IP). Una orientación fundamental de los nuevos estudios era la consideración biomédica de las materias incluidas en el plan de estudios, con lo que los estudiantes se formaban en los aspectos biomédicos desde el primer día del ingreso en el centro. Pero quizá la singularidad más importante de los estudios de Biología en la UPF es el quinto curso, dedicado al desarrollo de los itinerarios profesionales que se explican en la siguiente sección.

\section{CARACTERÍSTICAS GENERALES DE LOS ITINERARIOS}

Los IP se inspiraron inicialmente en el ejemplo del sexto curso rotatorio que se realiza en algunas facultades de Medicina (Jordi Camí, comunicación personal). En esencia, se trataba de que los estudiantes tuvieran una experiencia cuasi profesional antes de su graduación con el fin de promover la adquisición de las competencias que facilitaran su inserción profesional futura. Dada la orientación biomédica de la Facultad de Ciencias de la Salud y de la Vida de la UPF, tal experiencia se centró en aquellas áreas que, a juicio de los creadores del plan de estudios, concentraban la mayoría de las oportunidades laborales: investigación biomédica, laboratorio de análisis e industrias sanitarias.

Los IP se realizan en el quinto curso de la licenciatura y se estructuran en un primer trimestre dedicado a la formación específica en cada uno de ellos y dos trimestres con una inmersión completa de los estudiantes en centros de acogida con diferentes características (practicum). Durante el primer trimestre los estudiantes cursan asignaturas específicas (Tabla 1) siguiendo un modelo metodológico híbrido de la metodología de clase teórica con un $30 \%$ del tiempo dedicado a actividades de aprendizaje basado en problemas $(\mathrm{ABP})^{9}$. También realizan seminarios de discusión de artículos científicos en cada IP, así como una actividad conjunta llamada Biocinema ${ }^{10}$. Esta última consiste básicamente en la proyección de una película comercial (por ejemplo, Los niños del Brasil, Frankenstein de Mary Shelley, Estallido) seguido de un debate sobre los aspectos que se presentan en la película y que conciernen a la proyección social de la biología. Las actividades citadas ocupan las diez primeras semanas del trimestre.

La evaluación del curso se realiza mediante un examen de elección múltiple que supone un $45 \%$ de la calificación final, un análisis de un artículo cien- 


\section{Tabla 1. Asignaturas que se cursan en los itinerarios profesionales}

Itinerario de Industrias Sanitarias

- Desarrollo de nuevos fármacos y productos sanitarios (4,5 créditos)

- Farmacognosia y Tecnología farmacéutica (4,5 créditos)

Itinerario de Investigación Biomédica

- Patología molecular y celular (4,5 créditos)

- Patología molecular de sistemas (4,5 créditos)

Itinerario de Laboratorio de Análisis

- Bioquímica, Inmunología, Hematología y Genética clínicas (4,5 créditos)

- Microbiología y Parasitología clínicas y Análisis ambiental (4,5 créditos)

Asignaturas optativas (pueden cursarse por cualquiera de los alumnos de los tres itinerarios)

- Comunicación científica (4,5 créditos)

- Epidemiología general (4,5 créditos)

- Epistemología e Historia de la biología y de la medicina (4,5 créditos)

\section{Tabla 2. Los objetivos educativos del practicum del itinerario de Industrias Sanitarias}

Objetivos de conocimiento

a) Estructura de las industrias sanitarias.

b) Relaciones entre los diferentes departamentos.

c) Conocimientos básicos para la investigación y desarrollo (I+D) de nuevos fármacos y productos sanitarios.

d) Marco legal de los productos derivados de las industrias sanitarias.

e) Peculiaridades del ciclo de producción.

Objetivos metodológicos

a) Dinámica del trabajo en grupos.

b) Empleo de los medios informáticos que se utilizan en las empresas.

c) Elementos básicos de comunicación en las empresas.

d) Búsqueda de la información en situaciones reales.

e) Aplicación de los elementos de decisión a situaciones específicas.

Objetivos actitudinales

a) Primer contacto con el mundo de la empresa privada.

b) Relación interpersonal en el mundo laboral.

c) Limitaciones de la aplicabilidad de principios generales a situaciones determinadas.

d) Principios éticos de funcionamiento de las empresas.

e) Principio de cultura de empresa.

tífico (20\%), la resolución de un problema en formato ABP dentro del aula (20\%) y la evaluación de las actividades regulares de ABP (15\%).

En parte los IP constituyen una adaptación al Espacio Europeo de Educación Superior (EEES) y fueron concebidos cuando éste no se había dibujado aún en las declaraciones de la Sorbona y de Bolonia. En los tiempos agitados en que se están diseñando los programas oficiales de postgrado con sus másters y doctorados, los IP pueden considerarse en cierto modo un intento de adaptación al EEES avant la lettre. Sus objetivos principales se resumen en las tablas 2, 3 y 4 para cada IP pero, como puede observarse, pretenden acercar a los estudiantes al mundo real, tanto en el ámbito de la empresa como de los centros de investigación. En el primero ten- drían como objetivo la formación en un área de especialización profesional, mientras que en el segundo constituirían una iniciación a la investigación antes de la graduación. El hecho de realizarlos durante este período tiene claras ventajas para los estudiantes, pues les irresponsabiliza de sus resultados cuando aún no son licenciados y son estrechamente tutorizados durante este período. Es, utilizando un símil circense, como practicar equilibrismos con red de seguridad que protege si las cosas no funcionan adecuadamente.

Como puede observarse en las tablas citadas, los IP no pretenden dar una formación exhaustiva de los ámbitos que representan. Esto sería tarea vana debida su complejidad y la elevada especificidad de las tareas a realizar con frecuencia en el futuro en 


\section{Tabla 3. Los objetivos educativos del practicum del itinerario de Investigación Biomédica}

Objetivos de conocimiento

a) Estructura y funcionamiento del laboratorio de investigación biomédica, así como la distribución de tareas y responsabilidades del personal, en el entorno de centros de investigación básica (universidades, centros de investigación) o del ámbito sanitario (unidades de investigación en centros hospitalarios).

b) Base conceptual y práctica sobre tecnologías y sistemas experimentales empleado en los laboratorios de investigación biomédica.

\section{Objetivos metodológicos}

a) Dinámica del trabajo en grupo.

b) Aplicación del conocimiento adquirido a lo largo de la licenciatura al estudio experimental de los procesos biológicos en el laboratorio de investigación.

c) Habilidades de investigación, análisis y discriminación de la información especializada: uso de bases de datos bibliográfica y de otro tipo (secuencias génicas, proteínas).

d) Búsqueda de la información en una situación real.

e) Toma de decisiones en situaciones específicas.

f) Formulación de preguntas e hipótesis relevantes en el contexto de un proyecto de investigación y establecer conexiones con las otras áreas.

g) Priorización de objetivos y distribución de las fases de un proyecto de investigación.

Objetivos actitudinales

a) Funciones y responsabilidades del personal investigador y administrativo que compone un grupo de investigación.

b) Implicación y participación en el funcionamiento del grupo de trabajo.

c) Motivación hacia el esfuerzo individual propio sin detrimento de la capacidad de colaboración de los otros miembros del equipo.

d) Relación interpersonal en el laboratorio de investigación.

e) Principios éticos por los que debe regirse la investigación en el laboratorio.

f) Relación entre investigación básica y aplicada, comprensión del rol del investigador biomédico en la sociedad.

\section{Tabla 4. Los objetivos educativos del practicum del itinerario de Laboratorio de Análisis}

Objetivos de conocimiento

a) Estructura, funcionamiento, distribución de tareas y responsabilidades del personal dentro del laboratorio de análisis clínicos en el entorno relacionado con el ámbito sanitario (laboratorios privados de análisis o en hospitales e instituciones sanitarias).

b) Aplicación del conocimiento adquirido a lo largo de las diferentes asignaturas impartidas durante la licenciatura (bioquímica, microbiología, biología molecular, fisiología, fisiopatología, genética, histología, inmunología) al estudio de los procesos biológicos en el laboratorio de análisis clínicos.

c) Mecanismos por los que el organismo mantiene la homeostasis, causas de la variación biológica y de la fisiopatología asociada con las pruebas analíticas realizadas rutinariamente en el laboratorio de análisis clínicos.

d) Metodología analítica, indicación y selección diagnósticas, así como fuentes de error analítico.

e) Evaluación de los resultados analíticos y su interpretación clínica.

Objetivos metodológicos

a) Dinámica del trabajo en grupo.

b) Sólida base conceptual y práctica sobre tecnologías y sistemas experimentales empleados en los laboratorios de análisis clínicos.

c) Habilidades de investigación, análisis y discriminación de información especializada: uso de bases de datos bibliográficas y clínicas.

d) Aplicación de los conocimientos de búsqueda de la información en una situación real.

e) Toma de decisiones en situaciones específicas.

Objetivos actitudinales

a) Funciones y responsabilidades del personal que compone un laboratorio de análisis (técnicos de laboratorio, formación profesional, técnicos superiores, personal administrativo)

b) Implicación y participación en el funcionamiento del laboratorio.

c) Motivación hacia el esfuerzo individual sin detrimento de la capacidad de colaboración con el resto de miembros del equipo.

d) Características generales de la relación interpersonal del laboratorio.

el ámbito laboral. El objetivo de los IP es formar, en cambio, profesionales polivalentes capaces de adaptarse con facilidad a diferentes funciones gracias a conocimientos generales, desarrollo intenso de las competencias transversales y experiencia real e intensiva en los centros de trabajo.

\section{LA ESTRUCTURA DEL PRACTICUM}

Mientras los estudiantes cursan cuarto curso, se les informa de las características del quinto curso y se procede a la elección del IP que querían seguir en el año siguiente. Existía un número de plazas 


\section{Tabla 5. Empresas e instituciones públicas y privadas que han participado en el practicum de los itinerarios profesionales}

\section{Itinerario de Industrias Sanitarias}

Almirall-Prodesfarma, Amgen, Bayer, Biométrica, Boehringer-Ingelheim, CIDA, Crystax, Esteve, Farmacusí, Farmaprojects, Ferrer Internacional, Infociencia, Kern Pharma, KeyPoint, Lácer, Menarini, Noray Bioinformatics, Novartis Farmacèutica, Oryzon Genomics, Pan Lab, Prous, Rdes, Salvat.

\section{Itinerario de Investigación Biomédica}

Centre de Regulació Genòmica, Institut Municipal d'Investigació Mèdica, Universitat Pompeu Fabra, Institut de Recerca Oncològica, Institut Català d'Oncologia, Consejo Superior de Investigaciones Científicas, Parc Científic de Barcelona, Hospital Vall d'Hebron, Wurzburg Universität.

\section{Itinerario de Laboratorio de Análisis}

Institut Dexeus, Laboratori de Referència de Catalunya, Institut Municipal d'Investigació Mèdica, Hospital del Mar, Consejo Superior de Investigaciones Científicas, Hospital de la Creu Roja (I'Hospitalet de Llobregat), Laboratoris Balagué, Universidad de Chile.

limitadas en cada uno de ellos, con un máximo de 25 , por lo que los estudiantes deben elegir mediante la priorización de las tres opciones. La ordenación final se realiza en función de su expediente académico y si el número de solicitudes de un IP supera la cifra establecida, pasan a la segunda opción, aunque esta circunstancia no ha sucedido en ninguno de los tres años evaluados. Una vez establecido el IP que cursará cada alumno, se procede a escoger entre las distintas plazas para desarrollar las prácticas que ofrece cada itinerario (Tabla 5). En general, se ofertan alrededor de 1,5 opciones por alumno, de forma que las posibilidades de escoger son amplias. De nuevo, la elección se realiza por orden de notas, de manera que el mejor expediente de cada IP escoge primero y así sucesivamente. En el proceso de asignación, los estudiantes se entrevistan con los responsables de sus centros de acogida, de forma que unos y otros deben aceptarse mutuamente (proceso de mutua aceptación). Cada estudiante puede escoger entre dos opciones y debe decidir una de ellas antes de que el siguiente proceda a la elección.

Los estudiantes se incorporan a sus centros tras las vacaciones de Navidad y permanecen en él a dedicación completa hasta finales de junio. Durante este período de seis meses, los estudiantes deben incorporarse a la dinámica de los centros como un miembro más de los que allí trabajan. Para la evaluación realizan una memoria de fin de carrera supervisada por el tutor del centro de acogida y por el responsable de cada IP. La evaluación final del practicum se realiza por el informe del tutor, la presentación de la memoria de fin de carrera y la presentación de un póster en una sesión celebrada a finales de junio, en la que deben defender éste ante los asistentes, esencialmente profesores de la Facultad y tutores de los centros participantes.

\section{LOS RESULTADOS DE LA EXPERIENCIA}

Los resultados que se describen a continuación pertenecen a los tres primeros años de la experiencia, que corresponden a los cursos académicos 200203 y 2003-04. Siete años después de iniciarse la licenciatura en Biología en nuestra Universidad y con tres cohortes de egresados, es deseable analizar lo que ha sucedido con los cerca de doscientos graduados. Por esta razón, hemos realizado un seguimiento de su inserción laboral a fin de establecer si la estrategia de los IP ha contribuido en la mejora de la situación de indefinición profesional y ha mitigado la problemática del elevado paro o de la ocupación en profesiones alejadas de su formación universitaria.

La Tabla 6 describe la situación profesional de los 114 egresados de las dos cohortes de 2002-2003 y 2003-2004. Los datos de la cohorte 2004-2005 no se presentan pues, en el momento de la redacción de este artículo, sólo habían transcurrido tres meses desde su graduación y, por tanto, los resultados no son comparables a los de los egresados anteriores. La revisión de la Tabla 6 muestra como la gran mayoría de los egresados $(83,2 \%)$ se encuentran realizando actividades remuneradas, especialmente estudios de doctorado, muchos de ellos en centros externos a la Universitat Pompeu Fabra o sus institutos de investigación asociados (Institut Municipal d'Investigació Mèdica y Centre de Regulació Genòmica), aunque un número importante también se encuentran en otros organismos públicos (Universitat Autònoma de Barcelona, Universitat de Barcelona). Más de la cuar- 
ta parte (29\%) de los egresados trabajan en la actualidad en empresas biomédicas privadas o públicas, mientras que sólo cuatro graduados se encuentran realizando actividades no relacionadas con sus estudios. El análisis por itinerarios muestra que la mayoría que trabajan en empresas biomédicas del ámbito privado cursaron el itinerario de industrias sanitarias. De hecho, el 38\% de ellos trabaja en una empresa de estas características, mientras que otro $38 \%$ está realizando estudios de doctorado. Como era esperable, el $76 \%$ de los estudiantes del itinerario de investigación biomédica se encuentran realizando su tesis doctoral en centros públicos de investigación. En el caso del itinerario del laboratorio de análisis, el $33 \%$ se encuentran en empresas biomédicas o en centros públicos del ámbito sanitario y el $43 \%$ está realizando estudios de doctorado.

Otros resultados que merece destacar es que el número de estudiantes a la búsqueda activa de trabajo es muy reducida y, si se le suma aquellos de los que desconoce su situación profesional actual, el porcentaje llega al 6\%. También debe resaltarse que sólo un estudiante se encuentra en la docencia no universitaria, algo inesperado si se tiene en cuenta que es uno de las ocupaciones profesionales más tradicionales de los biólogos.

Tabla 6. Situación profesional de los egresados de las cohortes correspondientes a 2003 y 2004.

Empresas biomédicas Industrias sanitarias Investigación biomédica Laboratorio de análisis TOTAL

- Farmacéuticas
- CRO
- Comunicación médica
- Instrumentación

(1)

- Comunicación médica

- Instrumentación

$\begin{array}{rrrr}11 & - & 1 & 12 \\ 3 & - & - & 3 \\ 1 & - & - & 1 \\ 1 & - & 1 & 2 \\ & & & 18\end{array}$

Centros públicos del ámbito sanitario

- Hospitales

- Laboratorios de análisis clínicos no hospitalarios

- Otros

- Técnicos de investigación

$\begin{array}{lllr}3 & - & 4 & 7 \\ - & - & 4 & 4 \\ - & 1 & 1 & 2 \\ - & - & 2 & 2 \\ & & & 15\end{array}$

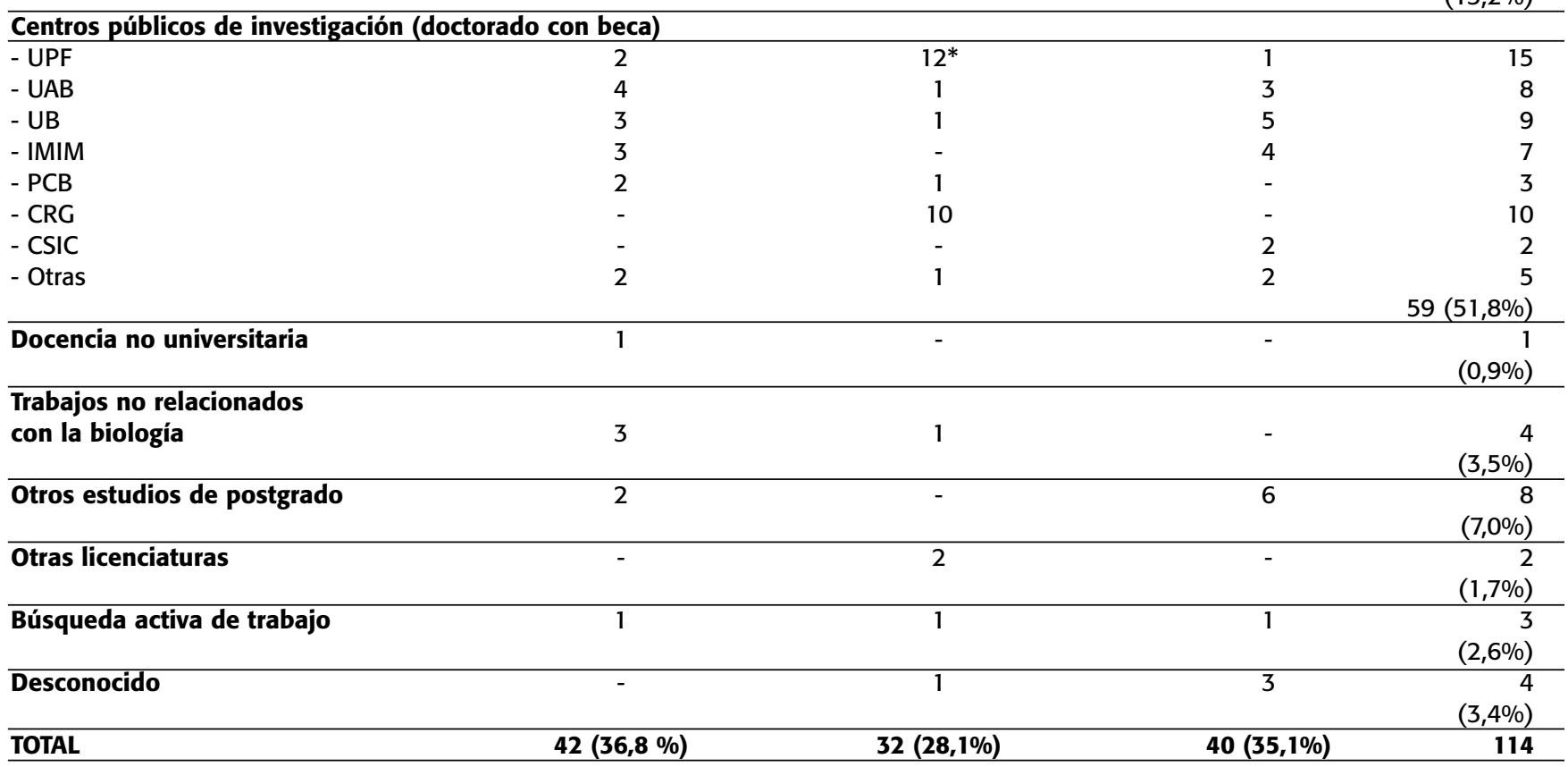

Abreviaturas. CRO: Empresa de investigación bajo contrato (Contract-research organization); UPF: Universitat Pompeu Fabra; UAB: Universitat Autònoma de Barcelona; UB: Universitat de Barcelona; IMIM: Institut Municipal d'Investigació Mèdica; PCB: Parc Científic de Barcelona; CRG: Centre de Regulació Genòmica; CSIC: Consejo Superior de Investigación Científica.

** Corresponden a unidades mixtas de investigación IMIM-UPF. 


\section{CONCLUSIONES Y REFLEXIONES FINALES}

En el año 2007 se graduará la quinta promoción de la licenciatura en Biología que se describe en el presente artículo. Con pocas modificaciones, estos estudiantes siguen el modelo de IP con el que se inició el quinto curso en el año 2002. Los responsables del centro consideraban como elemento esencial del éxito del plan de estudios la inserción laboral, consecuencia de la creación de un perfil profesional definido que situara a sus biólogos en el ámbito biosanitario de forma óptima. Los resultados presentados aquí son naturalmente la primera parte del proceso, ya que los próximos años mostrarán si esa mayoría de estudiantes que están realizando sus tesis doctorales encontrarán encaje en el sistema de investigación del nuestro y de otros países. Por tanto, el éxito de la licenciatura quedará reflejado en primer lugar por este hecho y en segundo lugar por el grado de inserción que las próximas cohortes de graduados obtengan. Es obvio que no podemos contestar por ahora a ninguna de las dos cuestiones.

Podría plantearse si el modelo de los IP expuesto es sólo una particularidad de nuestro centro o bien podría ser también exportable a otras universidades del entorno. Es obvio que el número reducido de estudiantes (aproximadamente 60 por curso) ha facilitado las cosas, aunque esta es condición necesaria pero no suficiente. Las promociones de gran número de estudiantes dificultan la realización de programas de estas características pero no los imposibilitan. Precisan de una infraestructura docente más compleja, pero que se encuentra presente en muchas universidades. Quizá el problema más importante es la realización del practicum pero sería recomendable que cada Universidad se adaptara a las posibilidades de su entorno para realizarlo. En el caso de los IP de Investigación Biomédica y de Laboratorio de Análisis, no existen mayores dificultades si se recluta a un número suficiente de investigadores y de profesionales de hospitales y centros sanitarios para que participen como tutores. En el caso de Industrias Sanitarias, es cierto que el entorno empresarial modula sustancialmente sus posibilidades. Así, una orientación hacia la industria farmacéutica es poco factible fuera del área metropolitana de Barcelona y de Madrid, pero existen en España más de cuarenta parques tecnológicos, muchos de ellos con empresas de investigación biotecnológica 0 bioinformática ${ }^{11}$, para considerar la posibilidad de que los estudiantes realicen allí su período de formación. En otras partes este itinerario podría orientarse, por ejemplo, a empresas agroalimentarias o a la formación de docentes de educación secundaria siguiendo el mismo modelo.

Debe comentarse también que la incorporación de los alumnos a los centros de investigación está adquiriendo amplia aceptación a través de estas primeras promociones. Ello puede ser debido a diversos motivos, pero destacaríamos como los más apreciados que el período de prácticas sea amplio y que la dedicación de los estudiantes sea exclusiva a esta labor durante dicho período.

Por último, desearíamos destacar el interés del modelo de IP para los programas oficiales de postgrado del EEES. Los IP permiten tanto la especialización profesional como la iniciación a la investigación y muestran cómo una parte de los estudiantes se inclina hacia los doctorados, pero una parte no menos importante obtiene un encaje profesional en los meses siguientes a la graduación. Es una experiencia que puede ser el punto de partida para la construcción de la nueva realidad del postgrado.

\section{AGRADECIMIENTOS}

Deseamos manifestar nuestro reconocimiento en primer lugar al profesor Jordi Camí, impulsor de la licenciatura en Biología de la Universitat Pompeu Fabra y que participó muy activamente en la concepción y desarrollo de los IP para darles la forma que tienen en la actualidad. En segundo lugar, a todos los investigadores y profesionales que a lo largo de estos años han atendido a nuestros estudiantes y les han ayudado a convertirse en profesionales de la biología. En tercer lugar, a todas las instituciones públicas y a las empresas privadas que creyeron en la experiencia y que sin las cuales jamás hubiera podido llevarse a cabo. En cuarto lugar, agradecemos a los estudiantes de estas promociones su apuesta por el modelo y la defensa diaria que han realizado de él con su trabajo diario en los diferentes centros de investigación donde han desarrollado las prácticas. $\mathrm{Y}$ en quinto, pero no en último, a la Generalitat de Catalunya por el reconocimiento de una iniciativa destinada a formar los profesionales que el sector biomédico de nuestro país necesita para no perder el tren de la sociedad del conocimiento y de la tecnología.

\section{BIBLIOGRAFÍA}

1. Reichert S, Tauch C. Trends IV: European Universities implementing Bologna. Brussels: European University Association, 2005. 
2. Gassiot Matas M. ¿Bolonia sí, Bolonia no? La Vanguardia, 29 de septiembre de 2005. pág. 27.

3. Prats J, Carreras J, Branda L, Miralles R, Fenoll MR, Rodríguez $\mathrm{S}$, Grifoll J. Competències professionals bàsiques comunes dels llicenciats en Medicina formats a les Universitats catalanes. Barcelona: AQU, 2004.

4. Oriol A, De Oleza R. Llibre blanc de les professions sanitàries a Catalunya. Barcelona: Departament de Sanitat i Seguretat Social, 2003.

5. Oriol A, Creus M. Llibre Blanc de les professions sanitaries. Àmbit infermeria. Barcelona: Institut d'Estudis de la Salud, 2003.

6. Erill S,Camprubí P. Contribución del biólogo a la farmacología en España. Monografías Dr. Antonio Esteve, 3. Barcelona: Fundación Dr. Antonio Esteve, 1987.
7. http://www.upf.es/estudiants/carreres/index.htm?opcio=2, consultada el 13 de noviembre de 2005.

8. Baños JE, Aramburu J, Bosch F, Pérez J, Sentí M. Enhancing pre-graduation contact with the real world: the experience of professional tracks in human biology students at University Pompeu Fabra of Barcelona. Educ Med 2004; 7:48.

9. Baños JE. El aprendizaje basado en problemas en los planes de estudio tradicionales: ¿una alternativa posible? Educ Med 2001; 4:4-12.

10. Baños JE, Aramburu J, Sentí M. Biocinema: the experience of using popular movies in the teaching of Biology. J Med Mov $2005 ; 1: 42-46$.

11. www.apte.org. Consultada el 13 de octubre de 2005. 